\title{
Quantifying radiation from thermal imaging of residential landscape elements ${ }^{\star}$
}

\author{
Jane Loveday $^{1,{ }^{*}}$, Grant Loveday ${ }^{2}$, Josh Byrne $^{1}$, Boon-lay Ong ${ }^{3}$, and Peter Newman ${ }^{1}$ \\ ${ }^{1}$ Curtin University Sustainability Policy Institute, Perth, Australia \\ 2 Independent Researcher, Perth, Australia \\ 3 Department of Architecture and Interior Architecture, Curtin University, Perth, Australia
}

Received: 15 January 2017 / Received in final form: 23 June 2017 / Accepted: 27 July 2017

\begin{abstract}
The microclimate of a residential landscape can affect both the energy use in your home and the human thermal comfort in your garden, ultimately affecting the heat in the neighbourhood or precinct. A thermal imaging camera provides information about the temperature of surfaces. By using Stefan-Boltzmann's law and the surface properties, these temperatures can be used to calculate the emission of longwave radiation (radiant exitance) in $\mathrm{W} \mathrm{m}^{-2}$. A thermal camera was used to determine the amount of radiant exitance from a range of residential landscape elements. A standard procedure for capturing these images was developed, taking into account factors which affect the quality of the radiometric data. A quantitative database comparing this radiation has been compiled for different times of day and different seasons. The sky view factor of these elements was chosen such that it was as close to 1 as possible. For a particular landscape design, areas of each landscape element can be measured and the amount of radiation reduced or emitted at different times can be calculated. This data can be used to improve landscape designs to reduce home energy use and human thermal comfort through shading and reduction of surfaces which emit longwave radiation close to the house.
\end{abstract}

\section{Introduction}

In the face of increased heat waves and the increase in city temperatures from the urban Heat Island effect, there is a need to reduce heat on both a meso- and micro-climate scale [1]. In the suburbs we can reduce heat entering houses and increase human thermal comfort in our gardens by looking to landscape design and the use of different surface treatments or landscape elements (LE).

LE such as trees, plants, and hard- and soft-scapes such as pavers and grass have different physical properties and thermal functions. These can either moderate the heat caused by incident solar radiation through either shading (reduction in both shortwave radiation (SWR) and longwave radiation (LWR) $[2,3]$ ), reflection or evapotranspiration, or can exacerbate the heat through absorption and re-radiation (radiant exitance) of LWR [4]. At night, elements can cool off through re-radiation to the cold night sky or can retain heat if thermal mass is high and other objects obscure their sky view (low sky view factor (SVF) [5]).

\footnotetext{
$\star$ This research is funded by the CRC for Low Carbon Living Ltd supported by the Cooperative Research Centres program, an Australian Government initiative.

* e-mail: jane.loveday@postgrad.curtin.edu.au
}

To quantify the radiative effects of different LE, a longwavelength infrared or thermal camera can be used to determine surface temperatures. Doulos et al. [6] studied 93 common outdoor pavement materials using a thermal camera and ultimately classified them into to "cold" and "warm" categories. Surface treatments studied were concrete, asphalt, marble, granite, pave stone, stone, pebble and mosaic. Doulos concluded that the main effect on surface temperature was due to the colour, surface texture and construction material. This paper extended the range of LE to include sands, soils and plants, as well as similar hardscapes. Samuels et al. [7] also used thermal imagery to measure temperatures of different LE. They determined the diurnal transient interactions between LE and ambient temperature in a particular urban location during summer and ranked them by hottest to coolest, creating a rule-of-thumb Thermal Performance Index; identifying elements as either radiators or coolers. They concluded that unless thermal energy can be transformed by vegetation, then it will continue to be an issue in the urban environment. This paper adds to this knowledge by measuring the radiant exitance (in $\mathrm{W} \mathrm{m}^{-2}$ ) from the $\mathrm{LE}$ under more controlled conditions, allowing a quantified comparison between LE both diurnally and over different seasons, without the influence of fluctuating radiant exitance from other urban elements. 
An accurate comparison of surface temperatures measured using a thermal camera across a range of LE is dependent on developing a consistent measurement and analysis procedure based on factors affecting the radiometric data, and on controlling the measurement environment as much as possible. Emissivity and reflected temperature are the most important factors to consider for temperature calculations within the camera [8]. Emissivity $(\varepsilon)$ is the fraction of LWR the surface emits compared with that from a black body, and for most LE it is around 0.95 .

Previous studies have only used the apparent temperature calculated by the camera software in order to compare surface temperatures. This is because accurate measurements of the emissivities and reflected temperatures are difficult in the field. These apparent temperatures do not include the reflected radiation from the LE, which in reality is still present and has an influence on objects around it. Surfaces with a lower $\varepsilon$ have a higher reflectivity and will therefore reflect a greater proportion of the surrounding background radiation. This becomes more significant for greater differences between the surrounding and the surface temperatures and is the reason that total radiation is more important to measure than apparent temperature.

For example, outdoors, the background radiation is dependent on the SVF which is the fraction of total sky "visible" to the LE. In a separate unpublished study by the authors, the individual emissivities of each of the LE were measured and determined to be greater than approximately 0.84 , standard deviation (SD) 0.02 , with an average $\varepsilon$ of 0.94 (SD 0.05). So, based on calculations using the StefanBoltzmann law and for surface temperatures $0-80^{\circ} \mathrm{C}$, for a $\mathrm{LE}$ with an $\varepsilon$ of 0.84 a change in reflected radiation from $-30^{\circ} \mathrm{C}$ (clear sky - high SVF) to $20^{\circ} \mathrm{C}$ (enclosed garden - low SVF) has been calculated to result in an increase of total (emitted + reflected) radiation of up to $12 \%$, depending on the temperature of the LE. However for a LE with $\varepsilon=0.95$, this change is only up to $3.5 \%$, again depending on the temperature of the LE. So radiation measurements from this study for the majority of LE (with $\varepsilon$ around 0.95 ) done at a high SVF, will be directly applicable for lower SVF situations. Hence this error only becomes significant when extending the results of the lower emissivity LE to a lower SVF situation. However the main aim here is to be able to compare radiant exitance from LE under controlled conditions.

This paper is about using a method to enable comparison of the measured LWR data across seasons and at varying temperatures, and applying this method to data measured over two seasons.

\section{Methodology}

\subsection{Study design}

The diurnal radiant exitance from a variety of different LE was measured. Although this has been done before for some of the LE, it is difficult to compare this flux density on different days even within the same season, as ambient temperature profiles vary from day to day. There is a need to find a way to normalise the data to allow this comparison. Other weather conditions can also affect radiant exitance from LE: wind conducts heat away (forced convection); irradiance from background ambient temperatures influence the LE; precipitation has an impact; and solar radiation is seasonal. To compensate for convection and precipitation, only measurements taken under average wind conditions and for no precipitation were compared. To compensate for diurnal temperature fluctuations, the data from each LE was compared to the LWR that would theoretically be emitted at the ambient temperature by a black body with $\varepsilon=1$.

Twenty-three types of surface treatments or LE were chosen, comprising those typically used in the Perth (Western Australian) metropolitan area (listed in Tab. 1). An open grassed area located on the Booragoon Primary School oval, Booragoon, Perth $\left(-32.035918^{\circ}, 115.826655^{\circ}\right)$, was chosen for the test work so as to give as large a SVF as possible. This was to reduce the variable radiant flux from any nearby landscape features due to changing solar exposure on them. The tests ran over 2-3 days during each of winter (11-13th July 2016) and spring (28-29th September 2016). LEs were placed directly onto to grass (in the case of concrete, limestone, all pavers and asphalt), on a breathable plastic bag in direct contact with the grass (white and black rocks) and slightly elevated from the grass on a breathable plastic bag placed on a plastic bread crate of dimensions $600 \mathrm{~mm} \times \mathrm{mm} 690 \mathrm{~mm} \times 150 \mathrm{~mm}$ (sands, soil and mulch). These placements were due to the nature of each LE and to the requirements of the land owner. Two 1$1.5 \mathrm{~m}$ high shrubs in pots (a ficus, non-native and a woolly bush, native) were also chosen, as well as two nearby large trees (10-20 $\mathrm{m}$ high, a native jarrah and non-native jacaranda). Note that the trees would have a different SVF to the other LE.

A commercial thermal imager (range 8-14 $\mu \mathrm{m}$ ) was used to take images of each LE every half hour from around 06:00 am to 20:00 pm. Images were taken from near-tovertical above each LE at a height of approximately $1 \mathrm{~m}$. A weather station was set up on the oval to record ambient temperature, relative humidity and wind speed and direction.

\subsection{Data analysis}

Only images from whole days of complete clear sky (in winter $13 / 07 / 2016$ and in spring 29/09/2016) were analysed using the camera manufacturer's software. The reflected temperature was measured during each set of images using crumpled aluminium foil and the reflector method [9]. In all cases this was below the lower limit of the thermal camera $\left(-20^{\circ} \mathrm{C}\right)$ and therefore assumed and set to be, $-30^{\circ} \mathrm{C}$ for all measurements. As explained above, the actual reflected temperature chosen makes little difference when the $\varepsilon$ is high. For the few LE with emissivities around 0.85 , the choice of the reflected temperature of the clear sky was also not significant (less than $2 \%$ effect) in changing from -20 to $-30^{\circ} \mathrm{C}$ ). However for these LE under much lower SVF conditions, the reflected component of the total radiation flux density becomes significant. 
Table 1. List of normalised longwave radiant energy per unit area for each LE. Data are sorted according to winter "Total".

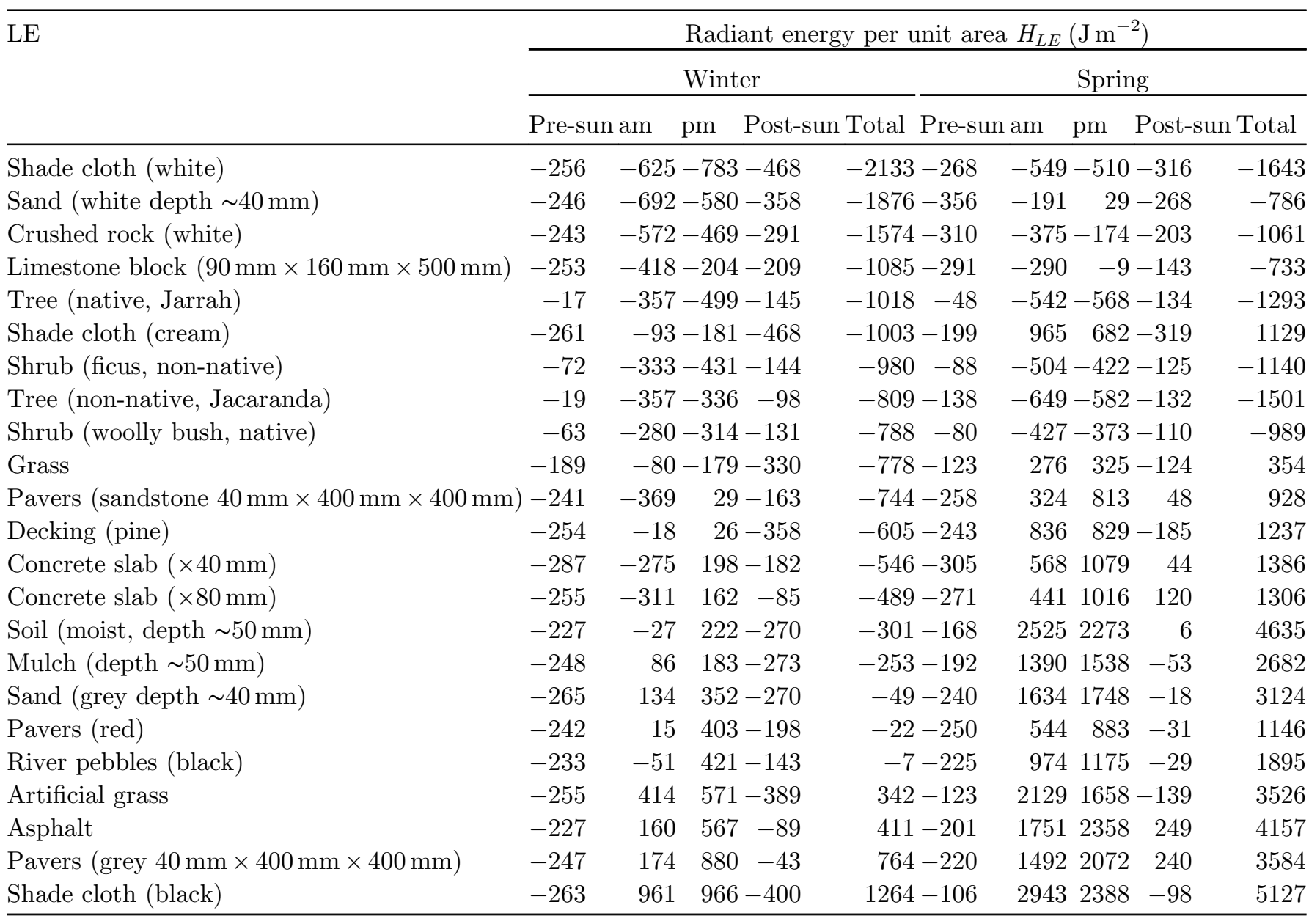

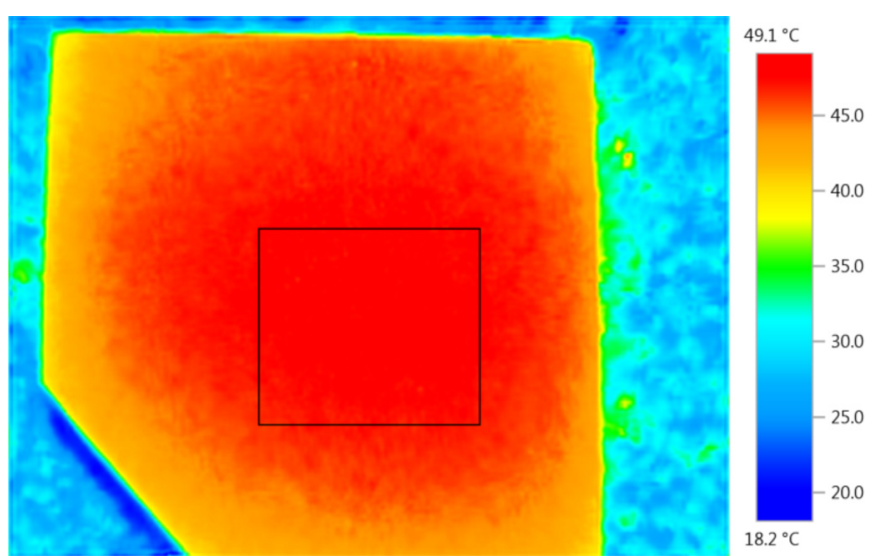

Fig. 1. Example of the thermal image of a grey paver with an averaging box.

Measured wind speeds $\left(<3.2 \mathrm{~m} \mathrm{~s}^{-1}\right.$ in July; $<2.72 \mathrm{~m} \mathrm{~s}^{-1}$ in September) were less than the average 9am and $3 \mathrm{pm}$ wind speed data from the Australian Bureau of Meteorology [10] for July and September.

An area box close to the centre of the landscape element image was manually drawn onto each image, and the average apparent temperature in this box was extracted (example Fig. 1).
The Stefan-Boltzmann law gives the amount of radiant exitance $M\left(\mathrm{~W} \mathrm{~m}^{-2}\right)$ from a body based on its temperature (Eq. (1)).

$$
M=\sigma \varepsilon T^{4},
$$

where $T$ is the absolute temperature of the body $(\mathrm{K})$ and $\sigma$ is the Stefan-Boltzmann constant $\left(5.67 \times 10^{-8} \mathrm{~W} \mathrm{~m}^{-2}\right.$ $\left.\mathrm{K}^{-4}\right)$.

At each measurement time the apparent temperatures were then converted into a total radiant flux density $M_{\text {total }}$ $\left(\mathrm{W} \mathrm{m}^{-2}\right)$, which is the sum of the radiant exitance from the LE $\left(M_{L E}\right)$ plus the reflected irradiance $E_{R E F}\left(\mathrm{~W} \mathrm{~m}^{-2}\right)$ and was calculated using equation (2):

$$
M_{\text {total }}=M_{L E}+E_{R E F}=\sigma \varepsilon T_{L E}^{4}+\sigma(1-\varepsilon) T_{R E F}^{4} .
$$

In order to normalise the $M_{\text {total }}$ across diurnal variations, the theoretical radiant exitance from a black body $M_{B B}\left(\mathrm{~W} \mathrm{~m}^{-2}\right)$ with $\varepsilon=1$ at ambient temperature (i.e. not in the sun), was subtracted from $M_{\text {total }}$. This produced a final radiant exitance $M_{\text {final }}$ for each LE at each measurement time (Eq. (3)).

$$
M_{\text {final }}=M_{\text {total }}-M_{B B},
$$




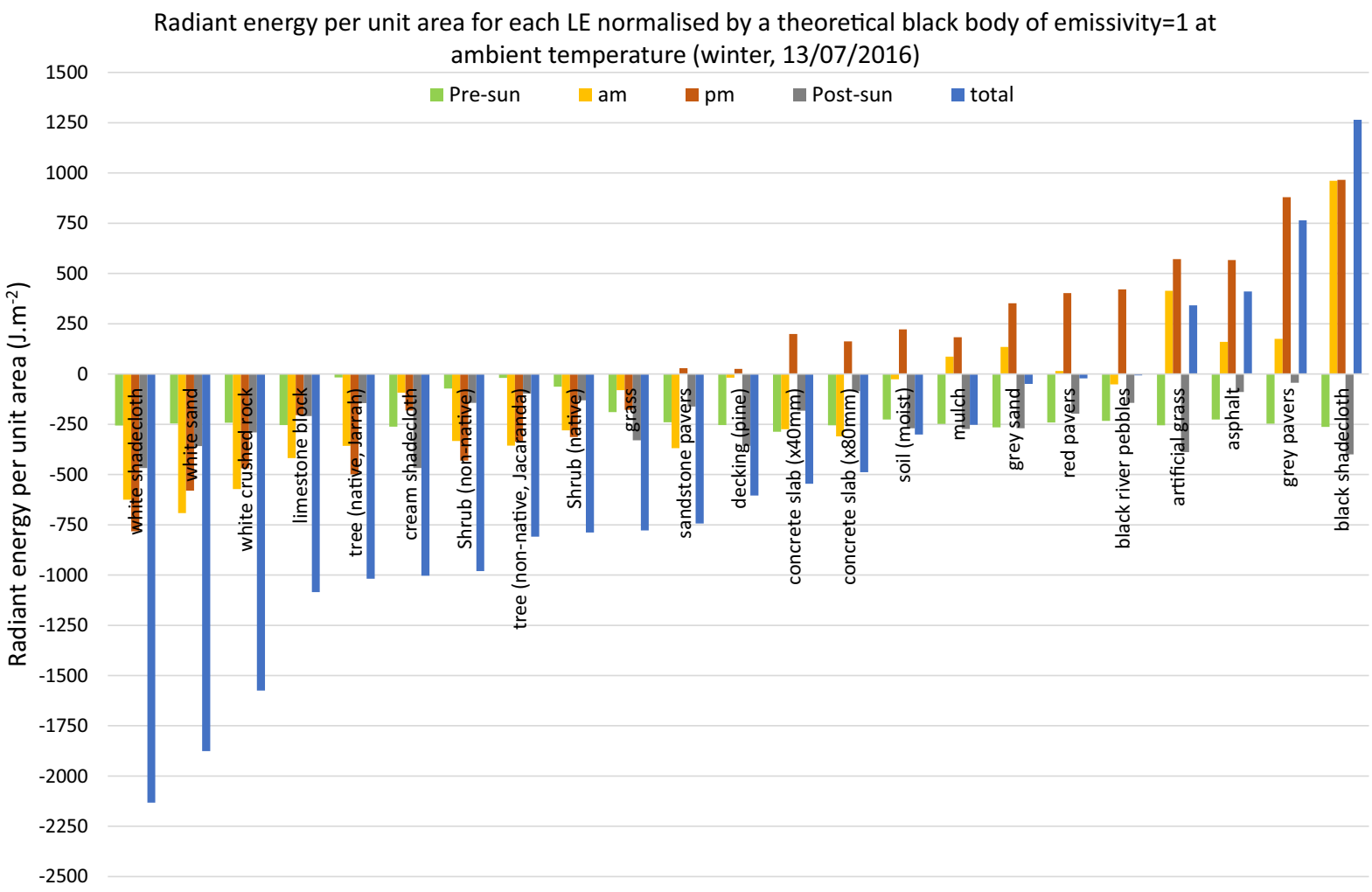

Fig. 2. Graph of final radiant energy per unit area $\left(\mathrm{J} \mathrm{m}^{-2}\right)$ for each LE in winter $(13 / 07 / 2016)$.

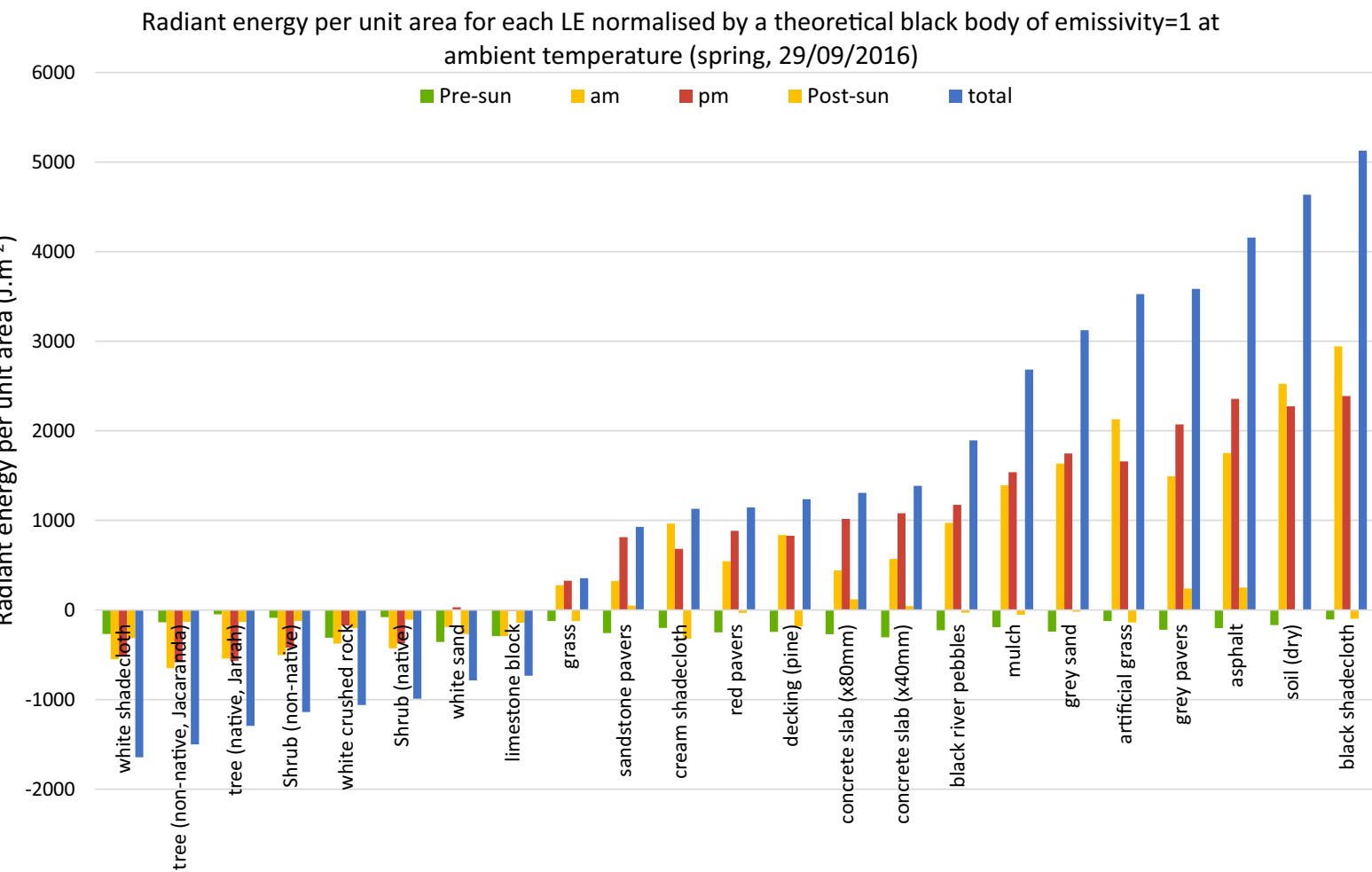

Fig. 3. Graph of final radiant energy per unit area $\left(\mathrm{J} \mathrm{m}^{-2}\right)$ for each LE in spring $(29 / 09 / 2016)$. 
$M_{\text {final }}$ for each LE were integrated over the measurement period to provide the total energy emitted by each LE surface (radiant energy per unit area $H_{L E}\left(\mathrm{~J} \mathrm{~m}^{-2}\right)$ ). To examine diurnal variation, $H_{L E}$ was divided into sections (noting that the LE were at equilibrium in the $2 \mathrm{~h}$ before and after their sun exposure): $2 \mathrm{~h}$ pre-sun on LE (Pre-sun); first sun on LE to solar midday $(a m)$; solar midday to last sun on LE $(p m)$; and $2 \mathrm{~h}$ post-sun (Post-sun). It was expected that the physical properties of the LE would affect how and when this energy was released and that this would be reflected in each section.

\section{Results}

A quantitative database comparing normalised radiant energy per unit area for each LE over two seasons is given in Table 1. For the winter and spring data, LE were first all in the sun at 08:00 am, whilst the last sun was at $16: 30 \mathrm{pm}$. Solar midday in Perth occurs at 12:30 pm.

These data are best represented graphically and are shown in Figures 2 and 3.

\section{Conclusions}

Half hourly thermal images of a number of different LE were taken over the period of a day during clear sky conditions and under a large SVF for two seasons, winter and spring. The radiant exitance was back calculated using the Stefan-Boltzmann law. The reflected radiation was included in the measurements as this is still present and has an influence on objects around it. The total exitance was normalised for diurnal temperature variations by subtracting the exitance from a theoretical black body of emissivity 1 at ambient temperature (i.e. not in the sun). This final exitance was integrated over the day and divided into four sections; $2 \mathrm{~h}$ pre-sun on LE; first sun on LE to solar midday; solar midday to last sun on LE; and $2 \mathrm{~h}$ post-sun.

As is known, the longwave radiant exitance of a $\mathrm{LE}$ is dependent on its physical properties, namely its thickness (mass), thermal conductivity and surface roughness. In the sun, the colour of an object will determine its ability to absorb SWR, thus leading to increased LWR exitance with increased darkness. This colour effect was evident for both seasons, where white and light coloured LE emitted much less LWR in total than darker and black LE and indeed maintained temperatures below ambient in winter (negative relative radiant exitance). However in spring, the greater quantity of both LWR and SWR meant that only the white LE were cooler than ambient temperature. It will be interesting to observe these effects in summer when radiation is at its peak.

The thickness of the LE and their ability to store heat internally is observed in the difference between the morning and afternoon values, with a higher difference indicating a thicker LE. For example, the am to pm differences for asphalt, grey pavers, concrete slab $(\times 80 \mathrm{~mm})$, concrete slab $(\times 40 \mathrm{~mm})$, and sandstone pavers are much higher than those of the other LE. However as colour is also important for the amount of absorbed energy, this difference is not a defining factor.
Apart from white LE, trees and shrubs show the smallest radiant exitance of all the LE despite their green colour. Plants cool through conduction to the atmosphere and through transpiration, which converts incident radiation into latent heat, maintaining their temperature close to ambient. This is apparent for both seasons.

The energy budget of a garden will be the same regardless of which types of LE are present: what comes in must go out. However it is when (daytime or night-time) and how it is released that is important (emitted, reflected, convected and conducted away, or transformed by vegetation into latent heat). For example, a thin black LE will not reflect in the visible spectrum and its temperature will rise rapidly, closely tracking the incoming radiation. Consequently, LWR exitance and convective losses to the atmosphere will closely track the incoming radiation. Compare this to a dark thick LE which again reflects almost nothing in the visible spectrum, but emits less LWR at the start of the day as most of the absorbed energy is conducted into the larger mass. However, it continues to emit for a longer period after maximum incoming radiation has occurred.

Something which has not been studied here is the short wave reflectance or albedo of the LE, which will need to be taken into account for situations where SVF is small such as in residential gardens. This is because while a white LE keeps itself cool by reflecting a high proportion of SWR, it will reflect this SWR onto nearby objects when SVF is low. This reflected SWR, unless it is transformed by plants, will cause nearby objects to heat up and their radiant exitance to increase. Thus during further test work over the remaining two seasons, the albedo will be measured.

Ultimately, quantifying the radiant exitance of all the LE present in a garden will enable averages for different landscape designs to be determined. This will provide a rating for that landscape which could be regulated and would lead to improved design with regards to urban heat.

\section{References}

1. W. Saman, J. Boland, S. Pullen, R. de Dear, V. Soebarto, W. Miller, B. Pocock, M. Belusko, F. Bruno, D. Whaley, J. Pockett, H. Bennetts, B. Ridley, J. Palmer, J. Zuo, T. Ma, N. Chileshe, N. Skinner, J. Chapman, N. Vujinovic, M. Walsh, C. Candido, M. Deuble, A Framework for Adaptation of Australian Households to Heat Waves (University of South Australia, Queensland University of Technology, University of Sydney, University of Adelaide, National Climate Change Adaptation Research Facility, Gold Coast, 2013), p. 242

2. A. Millward, M. Torchia, A. Laursen, L. Rothman, Vegetation placement for summer built surface temperature moderation in an urban microclimate, Environ. Manag. 53, 1043 (2014)

3. H. Akbari, M. Pomerantz, H. Taha, Cool surfaces and shade trees to reduce energy use and improve air quality in urban areas, Solar Energy 70, 295 (2001)

4. F. Lindberg, S. Onomura, C.S.B. Grimmond, Influence of ground surface characteristics on the mean radiant temperature in urban areas, Int. J. Biometeorol. 60, 1439 (2016) 
5. Y. Wang, H. Akbari, Effect of sky view factor on outdoor temperature and comfort in Montreal, Environ. Eng. Sci. 31, 272 (2014)

6. L. Doulos, M. Santamouris, I. Livada, Passive cooling of outdoor urban spaces. The role of materials, Solar Energy 77, 231 (2004)

7. R. Samuels, B. Randolph, P. Graham, T. McCormick, B. Pollard, Micro-Urban-Climatic Thermal Emissions: In a Medium-Density Residential Precinct (City Futures Research Centre, Faculty of the Built Environment, UNSW and HASSELL, Sydney, 2010)
8. R. Usamentiaga, P. Venegas, J. Guerediaga, L. Vega, J. Molleda, F.G. Bulnes, Infrared thermography for temperature measurement and non-destructive testing, Sensors 14, 12305 (2014)

9. ASTM-E1862, Standard Test Methods for Measuring and Compensating for Reflected Temperature Using Infrared Imaging Radiometers (American Society for Testing and Materials, Pennsylvania, 1998)

10. BoM, Bureau of Meteorology climate statistics 1993-present (2017)

Cite this article as: Jane Loveday, Grant Loveday, Josh Byrne, Boon-lay Ong, Peter Newman, Quantifying radiation from thermal imaging of residential landscape elements, Renew. Energy Environ. Sustain. 2, 17 (2017) 